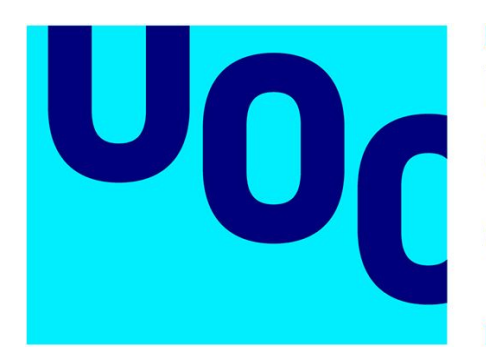

Universitat

Oberta

de Catalunya

\title{
Previsions i realitats de la Intel-ligència Artificial. Evolució 2015-2017
}
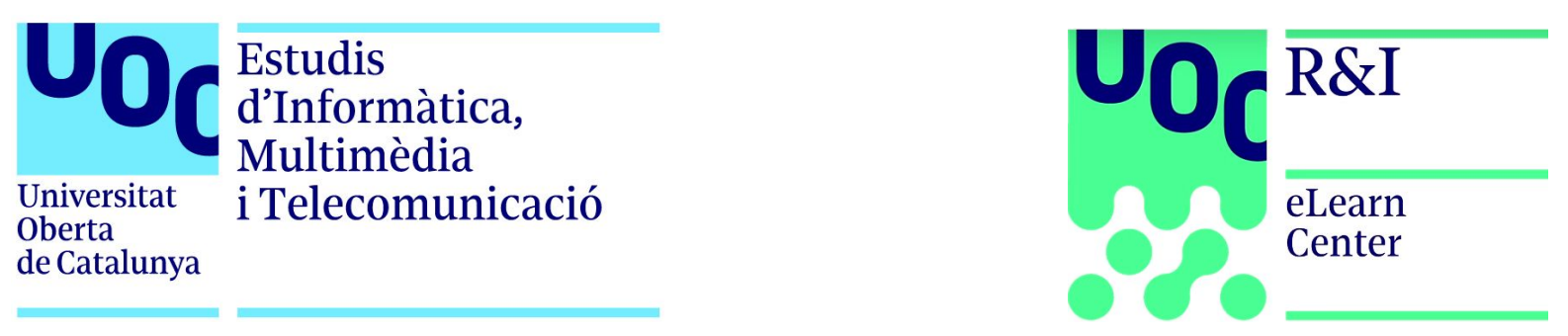


\section{Previsions i realitats de la Intel-ligència Artificial. Evolució 2015-2017}

\section{Crèdits}

El present informe és una iniciativa dels Estudis d'Informàtica, Multimèdia i Telecomunicació i l' eLearn Center de la Universitat Oberta de Catalunya.

Coordinadors: Guillem Garcia Brustenga, Lluís Pastor Pérez, Josep Prieto, Antoni Pérez Navarro, Eugènia Santamaria.

Autors: Robert Clarisó Viladrosa, César Pablo Córcoles Briongos, Desirée Gómez Cardosa, Josep Jorba Esteve, Enric Mor, Antoni Pérez Navarro, Laura Vergoñós Pascual i Irma Vilà Òdena.

Edició: Desirée Gómez Cardosa i Laura Vergoñós Pascual.

Reconeixements: Marta Bernabeu Torrecilla, Marc Fuertes Alpiste, Roger Griset Miró, Núria Molas Castells, José López Ruiz.

Citació APA recomanada: eLearn Center (2018). Previsions i realitats de la Intel-ligència Artificial. Evolució 2015-2017. Barcelona: eLearn Center (UOC).

ISBN: 978-84-09-04217-3

DOI: $\underline{\text { https://doi.org/10.7238/elc.ai.2018 }}$

\section{Llicència}

Atribució Creative Commons 4.0 (CC BY 4.0): https://creativecommons.org/licenses/by/4.0/ Repliqui, copiï, distribueixi, transmeti o adapti aquest informe lliurement sempre i quan es proveeixi l'atribució com es mostra a la citació.

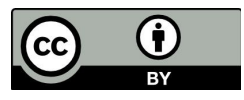




\section{Foreword}

Des dels Estudis d'Informàtica, Multimèdia i Telecomunicació (EIMT) es va engegar el 2014 l'Observatori Tecnològic (OTEIMT), amb l'objectiu de saber quines són les tendències en els àmbits d'interès dels Estudis. L'observatori es va constituir amb representants d'informàtica, de multimèdia, de telecomunicació i d'art (des de la vessant digital), però a més, com que el nucli de la tasca dels EIMT és l'e-Learning d'aquestes disciplines, I'OTEIMT treballa en colllaboració amb l'eLearn Center, que també hi està representat.

En els diversos informes i actes de reunió de l'OTEIMT hi ha hagut un tema que ha anat adquirint una especial rellevància, i tot apunta que al llarg dels propers anys la seva importància anirà en augment: la intel-ligència artificial (IA). Però, per què ha esdevingut tan important? Quins canvis s'han produït que l'han situat en el centre del debat, no només tecnològic, sinó també social? Afectarà la nostra vida quotidiana? Analitzar l'evolució de la IA des de la perspectiva de l'OTEIMT pot ajudar a respondre aquestes i altres preguntes, i això és, precisament, el que fa el present document.

Antoni Pérez Navarro

Sots-director de Recerca de l'eLearn Center

Estudis d'Informàtica, Multimèdia i Telecomunicació

L'eLearn Center, mitjançant el grup de Recerca i Anàlisi de Tendències, té com a objectiu detectar i monitoritzar les tendències pedagògiques, tecnològiques i socials que poden afectar a la UOC a mitjà termini, analitzar-les i fer-ne difusió per tal de posicionar la UOC i l'eLearn Center com a referents en el camp de l'e-Learning. Per tal d' assolir aquest objectiu fem tres activitats clau: observar, analitzar i difondre. Analitzar el que observem és imprescindible per posar en valor i complementar les accions més immediates de divulgació, opinió i curació de continguts. El que donarà rellevància a la feina que fem serà aquesta difusió de coneixement basada en l'anàlisi rigorós, tant cap al públic especialitzat com al públic general. És dins aquest marc on s'ha d'encabir la col-laboració de l'eLearn Center amb els Estudis d'Informàtica Multimèdia i Telecomunicacions i el seu Observatori Tecnològic.

La Intel-ligència Artificial és un dels temes analitzats a l'Observatori dels Estudis en els darrers anys, i és un tema del qual actualment se'n parla molt als mitjans generalistes i genera un certa curiositat social. En la IA, i en els xatbots en particular, hi veiem moltes possibilitats educatives a mig termini i per tant, també és d'alt interès per a l'eLearn Center. És per aquesta raó que ens ha semblat molt indicat fer aquest informe de Previsions i realitats de la Intel-ligència Artificial. Evolució 2015-2017.

Guillem Garcia Brustenga

Director del grup de Recerca i Anàlisi de Tendències eLearn Center 


\section{Introducció}

L'English Oxford Living Dictionary defineix la Intel-ligència Artificial (en endavant, IA) com la teoria i el desenvolupament de sistemes informàtics capaços de realitzar tasques que normalment requereixen intel-ligència humana, com ara la percepció visual, el reconeixement de la parla, la presa de decisions i la traducció entre idiomes [63]. El terme va ser utilitzat per primer cop per John McCarthy el 1955 dins la proposta d'estudi Dartmouth AI Project Proposal [64].

En referència a aquesta definició ens hauríem de qüestionar si estem demanant massa a la Intel-ligència Artificial. Som poc realistes en les prediccions del que pot esdevenir? Segons Roger Penrose [65] la consciència humana no és algorísmica i, per tant, no és capaç de ser modelada per una màquina de Turing convencional, com ara una computadora digital. Penrose considera que la mecànica quàntica haurà de jugar un paper essencial en la comprensió de la consciència humana i, en conseqüència, en la intel-ligència artificial.

Els avenços que semblaven inimaginables fa uns anys s'estan manifestant a través del camí traçat pel progrés tècnic. Actualment, aquesta tecnologia ja facilita algunes tasques diàries, millora el rendiment, detecta malalties en organismes o anomalies dins de processos mecanitzats o informàtics, capta dades per a la realització d'estadístiques, fa fotografies d'alta qualitat, i així fins a una gran quantitat de tasques i activitats que fins ara eren domini només dels éssers humans.

Tot $\mathrm{i}$ aquests èxits, hi ha àmbits en els quals encara s'està lluny de les expectatives que s'havien creat. Un exemple seria l'ús de Watson d'IBM [66] per recomanar tractaments per al càncer, de la qual s'esperaven grans resultats; tanmateix, aquesta IA encara està en fase d'aprenentatge de les diferents tipologies de la malaltia, així que la seva implementació no ha resultat ni tan ràpida ni tan eficient com en un principi es creia que podria ser [66].

D'altra banda, tot i que fa anys que imaginem com podrien ser les tecnologies derivades de la intel-ligència artificial, a mesura que han anat esdevenint realitat, han anat sorgint dubtes tant a nivell ètic com funcional.

Els xatbots han donat pas a converses amb un to cada cop més humanitzat amb les màquines. La tecnologia està cada cop més relacionada amb l'àmbit de la salut i sorgeixen dubtes sobre la condició transhumana; la seguretat es relaciona amb la vigilància i fins i tot l'anticipació dels crims; els robots podrien ocupar els llocs de treball de milers de persones que podrien quedar relegades a un segon pla i així un llarg etcètera que ens duu a plantejar-nos la regulació de tots aquests avenços. 
Des de l'Observatori Tecnològic dels Estudis d'Informàtica, Multimèdia i Telecomunicació, en colllaboració amb l'eLearn Center, s'ha volgut fer un recull en matèria d'Intel-ligència Artificial de les previsions en contrast amb les realitats que s'hi han anat manifestant des de l'any 2015 fins el 2017.

L'ànim ha estat veure, d'una banda, quina ha estat la seva evolució i com ha impactat en les nostres vides; i de l'altra, fins a quin punt s'han fet realitat, o no, els pronòstics que se n'havien fet. Per fer aquest informe, s'ha recollit la informació de dues fonts principals: d'una banda, les previsions de tendències de mitjans especialitzats i de l'altra, les notícies i informes de fonts més o menys divulgatives de l'àmbit.

El document s'estructura per anys. Per a cada any es mostra quines eren les previsions i quina en va ser la realitat, pel que fa a l'aplicació de la IA en diferents àmbits. Al final s'inclou una infografia de resum i un apartat de conclusions.

\section{Previsions del 2015}

Les bases de la IA van ser establertes entre les dècades dels 70, 80 i 90, i la major part dels avenços tècnics i algorítmics relacionats amb ella daten d'aleshores [69] [70]. Tot i això, l'actual tecnologia està fent possible el que s'havia dissenyat conceptualment [71]. Tanmateix, a inicis de l'any 2015 la IA no semblava ser una tendència principal segons especialistes com ara Frog [1], IEEE [2], Horowitz [3], MIT [4] i CTecno [5].

Una de les institucions que més s'hi va aproximar va ser Gartner [6]. Tot i així, en lloc de parlar d'IA, parlava d'aspectes tecnològics vinculats a la IA en les seves conclusions, com ara el fet que les màquines jugaran un paper cada cop més important en la presa de decisions.

D'altra banda, Usbek \& Rica, el 2015, cita l'estudi de BCC Research [60] on es menciona que el segment dels robots autònoms, incloent vehicles per a l'exploració espacial, era valorat en 1.1 bilions de dòlars el 2013 i estimava que serà de prop de 3.6 bilions de dòlars el 2019 i de 13.9 el 2024. Segons l'estudi, el mercat global per a les màquines intel-ligents augmentarà fins a un total de 15.3 bilions de dòlars el 2019, amb un creixement anual d'un $19.7 \%$, la qual cosa visibilitza en xifres la importància que tindrà la implementació de la IA en diferents mercats. Usbek \& Rica asseguren que el futur de la IA es troba en mans de quatre grans empreses [61]: Google (Nest Labs, Deep Mind, Dark Blue Labs, Vision Factory); Apple (Metaio, Vocal IQ); Facebook i Amazon. Altres gegants de la IA serien IBM, Baidu, Microsoft i Yahoo. A banda, destacava com a aplicacions principals la medicina, l'educació, la llar, el camp laboral, la mobilitat i els assistents personals. 


\section{Realitat l'any 2015}

Com s'ha dit anteriorment, les bases de la IA són de les dècades dels 40-50 i van ser desenvolupats en gran part les dècades dels 80-90. Però és a partir del 2006 quan algunes d'aquestes teories s'han pogut implementar gràcies a que s'ha disposat de prou potència de càlcul per poder-los aplicar. En gran part els desenvolupaments més útils es van donar en arquitectura de CPUs i GPUs (targetes gràfiques) ${ }^{1}$.

El 2015 ja es veuen les traces d'on pot derivar i dos dels aspectes més rellevants d'aquell any van ser:

- En primer lloc, una carta oberta [7] per prohibir el desenvolupament i l'ús d'armes autònomes signada per Hawking, Musk i Wozniak juntament amb 3000 investigadors en IA i robòtica.

- En segon lloc, l'AlphaGo de Google Deep Mind ${ }^{2}$ va vèncer tres vegades al jugador professional Fan Hui, campió del European Go, per 5 partits a 0 [8].

D'altra banda, mitjans com ara Future of Life Institute van fer un recull d'innovacions en IA, entre les quals va destacar, entre d'altres, el pensament abstracte creatiu [56]. Bloomberg va posar el 2015 com a any trencador per a la IA gràcies a les xarxes neuronals i els grans avenços en informàtica al núvol (cloud computing) [57]. Per la seva banda, el MIT Technologic Review [58] ressenya el 2015 com l'any en el qual els cotxes autònoms van esdevenir una realitat comercial. El 2015 també va ser també l'any de la competició de robots DARPA Robotics Challenge a Pomona, Califòrnia[59].

\section{Previsions el 2016}

Durant el 2016, la IA agafa empenta i es col-loca en una posició principal dins la I+D. Aquest any, entra ja dins les previsions de les principals entitats de referència com a àmbit a tenir en compte i camp en el qual dedicar-hi esforços: Google inclou la IA dins les seves 6 tecnologies que canviaran el món [9]; a l'informe de l'IEEE per al 2022 [10] ja es parla obertament del Machine Learning i dels sistemes intel-ligents; segons PEW [11], la vida digital al 2025 també mostra una previsió de tendències als sistemes smart i la interconnexió en un ambient global i immersiu; en el "ClO New Year's Resolutions 2016" [12], la IA es considera una tecnologia emergent puntera sobre la qual cal estar-ne al dia; $i$ amb una visió més enllà del moment de la publicació, Forrester's [13] també l'assenyala com a tecnologia capdavantera de cara al 2017.

\footnotetext{
${ }^{1}$ Veure notes [69][70] i[71].

${ }^{2}$ Versió Fan.
} 


\section{Realitat l'any 2016}

Durant el 2016, la IA va guanyar presència arreu, però apareix lligada, sobretot, a la robòtica i als Smart Cars o cotxes intel-ligents. Va haver un avenç gegantí i sistemes d'IA es van anar incorporant a la vida quotidiana gairebé sense que en fóssim del tot conscients. Un exemple és la convivència humana amb assistents com ara Siri d'Apple, Google Assistant, Alexa d'Amazon o Cortana de Microsoft, el traductor de Google o els sistemes TAC i de raigs $X$ que analitzen les dades obtingudes del nostre cos per detectar possibles tumors.

La investigació i els ràpids avenços d'un sector amb un potencial grandiós disparen inevitablement certa alarma social sobre l'hipotètic futur que es presenta. Dins una vessant alarmista lligada a la progressiva inclusió de la IA dins els àmbits de la vida quotidiana, han sorgit múltiples notícies sobre la possibilitat que els robots puguin fer la majoria de tasques que actualment fan les persones, i puguin substituir els humans amb major eficiència en alguns llocs de treball i generar una elevada taxa d'atur. Aquesta visió va derivar en una alarma social sobre el futur de la nostra vida laboral, sobretot quan parlem de les feines poc qualificades i mecàniques, que serien les més afectades. Els titulars d'alguns articles van ajudar a transmetre la idea que les persones siguin substituïdes per robots a la feina, com ara "La meitat de la població mundial podria quedar a l'atur a causa dels robots" [14] o "Un bot aconsegueix recórrer multes de trànsit per valor de 3 milions de dòlars" [15] en referència a altres tasques quotidianes com ara la resolució de tràmits burocràtics.

En aquest sentit, el debat s'hauria de centrar en un ajustament a la nova realitat tecnològica, on potser sí que algunes professions podrien quedar obsoletes per una major eficiència de les màquines, però on també naixerien noves professions lligades a aquesta realitat actualitzada [16]. Alguns exemples d'aquests nous llocs de treball podrien ser: mecànic de cotxes intel-ligents, especialista en transport autònom, intèrpret mèdic 0 , fins $\mathrm{i}$ tot, entrenador de xatbots, entre molts altres.

Dins aquest corrent preocupat per una evolució cap a la vessant negativa de la IA, el Future of Humanity Institute, creat per analitzar possibles riscos per a la humanitat en general, va incloure la IA dins el seu llistat [17]; i figures importants del món de la ciència van demanar la prohibició sobre el desenvolupament d'armes intel-ligents, ja que l'autoregulació no es considera suficient per impedir el seu desenvolupament [18].

Un focus on s'haurà de parar especial atenció és l'evolució de la IA en relació a la interacció humà - màquina i com la màquina "aprèn" dels humans. Primer de tot, les màquines han de conèixer i saber com són els humans. Facebook està experimentant en aquesta línia a través de clàssics de ficció infantil per a alimentar la seva IA [19]. 
En el cas de l'aprenentatge de la IA a través de la interacció amb persones, en alguns casos, pot haver-hi problemes, com va passar amb Tay, la IA de Microsoft [20] que va incorporar llenguatge groller i un discurs xenòfob a causa del que alguns adolescents van ensenyar-li. Altres paranys a la IA s'han donat també gràcies a l'enginy i picaresca dels que han creat marcs d'ulleres que enganyen els algoritmes de Machine Learning de manera que interpreten que la persona és una celebrity [21]. Una IA pot patir (com altres programes algorísmics) vulnerabilitats i possibilitats de hackeig o tergiversació d'informació per tal de produir resultats diferents dels que hauria d'obtenir [67].

Relacionat amb la interacció humà-màquina, s'han trobat casos de persones que arribarien a mentir als robots per evitar ferir els seus sentiments [22]. Aquesta capacitat de l'ésser humà per "humanitzar" coses és un punt d'especial preocupació en temes de robòtica i Machine Learning. Això es veurà més clarament durant l'any 2017, on es reflexiona més profundament sobre els aspectes ètics que aquesta tecnologia ens planteja i que es veu reflectit en l'Informe OTEIMT 2017 [23], sobretot quan va lligat a la robòtica humanoide.

El 2016 també va haver un elevat número de fites assolides per la IA: una IA japonesa va passar el primer filtre d'un certamen literari real [24]; la IA Watson desenvolupada per IBM DeepQA, va ser capaç de respondre en un llenguatge natural i es va posar a treballar com a conserge en un hotel (Pebble Beach Company); a la Georgia Tech University, el professor Ashok Goel va utilitzar també Watson d'IBM com a assistent sota el nom de professor Jill Watson; i també va veure la llum el projecte Say What You See de Facebook [26] que genera descripcions parlades de les fotografies a través d'una IA.

El 2016 va seguir havent força eco en premsa sobre la derrota del campió humà de Go a mans del software de Google ${ }^{3}$. Cal destacar que la capacitat del maquinari encara és molt limitada quan la comparem amb la mida de l'arbre de jugades potencial, per la qual cosa cal assumir que aquest programari és molt més sofisticat que el que va derrotar Kasparov en el seu moment [27]. Pel que fa a l'aprenentatge, les evolucions de la IA oscillen del rol d'"estudiant" al de "professor", com en el cas del robot centpeus que ensenyarà els robots com caminar [28].

Pel que fa al debat ètic, molt lligat a les preocupacions abans esmentades, s'ha iniciat un corrent "filosòfic" on hi ha experts que es comencen a fer preguntes, com ara: cal entendre els algoritmes que provenen de xarxes neuronals i dels quals, per tant, no en podem explicar completament el seu funcionament? Cal regular-los? Calen unes lleis de la robòtica de l'estil de les que va proposar l'Isaac Asimov? [29]. Potser la pregunta és més aviat una altra: una IA que utilitzi aquestes tècniques es pot arribar a explicar? Hauríem de saber per què ha "raonat" d'una determinada manera o quin ha estat el seu procés per arribar a la seva decisió. Si no es pot arribar a fer això, el mètode d'aprenentatge utilitzat seria

\footnotetext{
${ }^{3}$ Veure referència [8]
} 
qüestionable i portaria a dubtar sobre fins a quin punt ha estat manipulat per donar la resposta final.

Algunes de les bases que es podrien establir per a la regulació de tota IA són les següents:

- Ha de ser dissenyada per ajudar a la humanitat.

- Ha de ser transparent.

- Ha de maximitzar l'eficiència sense destruir la dignitat de les persones.

- Ha de ser dissenyada per a la privacitat intel-ligent.

- Ha de tenir responsabilitat algorítmica.

- Ha d'evitar el biaix.

En general, la IA està posant les bases d'un xoc a Europa [30] i hi ha ordinadors que no acabem d'entendre què fan [31]. En una ponència d'Hiroshi Ishiguro (Osaka University, Japó) sobre societats robòtiques, es menciona que els robots no necessiten entendre les persones per interactuar amb elles. Parlen sobre alguns trucs d'aquests per comunicar-se amb les persones, com ara canviar de tema, ignorar la resposta, etc. Tot i això, cal dir que els algoritmes podrien ser més fiables que els humans [32]: tot i que el sistema predictiu de la política de Chicago va fracassar en un test [33], una IA aplicada a sistemes mèdics va triomfar ja que un "metge" basat en IA guanya en diagnòstic visual perquè és capaç de veure diverses vegades més senyals de càncer de pulmó [34]. Tot i així, hi ha moltes crítiques en aquest sentit. A tall d'exemple, en els sistemes d'ajuda a la diagnosi, alguns sistemes han estat acusats de tenir un cert biaix cap a tractaments específics que no sempre són els millors [68].

A mig camí entre la IA i altres àmbits, també cal esmentar els esforços per dur la IA del "gran hardware" als dispositius mòbils (entesos en sentit prou ampli): xarxes neuronals als cotxes autònoms, smartphones i drones [25]. Tot i això, una combinació de mòbil i Cloud ho podria solucionar, com fan, per exemple els assistents per a mòbils de Google, Apple i Microsoft.

\section{Previsions el 2017}

A inicis de 2017, pel que fa a les previsions que realitzen entitats de pes dins el món tecnològic com ara Gartner [35] o l'IEEE [36], ja era una qüestió indiscutible que s'havia de parar especial atenció a la IA i tota la tecnologia relacionada. Des de les que permeten estructurar-la com l'aprenentatge automàtic i Deep Learning a les tecnologies que se'n deriven de la seva aplicació: robòtica, IoT, etc. L' IEEE afirma que hi ha un "Avenç de la IA i el Deep Learning fins a límits on no havien arribat abans" (Gartner 2017). 
El mitjà Futurism recull en una elegant infografia d'estil vintage [62] una previsió de la IA per al 2017 que beu, entre d'altres, de les fonts del MIT Tech Review i de CIO i que resumeix les opinions més esteses de l'any. Destaquen les següents previsions:

- Hi haurà més interfícies conversacionals (xatbots i d'altres).

- S'incrementarà el nivell de confiança en la IA.

- S'establiran estàndards universals d'IA.

- S'aplicarà la IA en més Apps i serveis.

- Microsoft i Apple faran servir Cortana i Siri a la manera de l'Echo ${ }^{4}$ d'Amazon (basat en l'assistent Alexa).

- Es farà servir el Deep Reinforcement Learning (emprat en el triomf al joc Go per part de Google contra un humà) per a enginys com ara els cotxes autònoms, els robots industrials, etc.

- Es desenvoluparà IA per a crear models predictius amb Big Data per a noves Apps i serveis.

- Es reconeixerà la $\mathrm{IA}$ com a intel-ligència augmentada (humans i màquines complementant les seves capacitats).

- Es treballarà en l'ètica relacionada amb la IA.

- Es farà servir la IA en negocis majoritaris.

\section{Realitat del 2017}

Si durant el 2016 la IA es va anar incorporant a les nostres vides (sobretot de manera incipient en aspectes relacionats a la robòtica o en cotxes intel-ligents), el 2017 es podria considerar l'any del desplegament a una escala considerable de la IA. Fent una analogia amb les xarxes neuronals, podem dir que la xarxa de la IA s'ha començat a "expandir $\mathrm{i}$ ramificar".

Aquesta expansió es pot palpar en l'aparició de projectes col·laboratius com ara el PAIR de Google (People + Al Research). A part dels propis treballadors de Google, en ell hi treballen, Hal Abelson i Brendan Meade del MIT i Harvard, respectivament. Es busca aconseguir millorar les capacitats dels sistemes d'IA per tal de fer-los més útils a l'hora de satisfer les necessitats de les persones. L'objectiu és "estudiar i redissenyar les formes en què les persones interactuen amb la IA" per aconseguir que la tecnologia "beneficiï i capaciti a tothom" per conèixer com millorar educació i ciència mitjançant la IA [37].

Tot i això, aquest desplegament es realitza moltes vegades a nivell experimental encara. L'ús real pràctic, que convisqui amb l'usuari diàriament, encara és força incipient; l'exemple més clar seria Facebook i l'API.ai per a l'expansió de l'espai personal. $\underline{H o w d y}$, X.ai, $\underline{\text { Clara i }}$

\footnotetext{
${ }^{4}$ Caldria afegir-hi Google Home, competidor directe d'Echo.
} 
Kasisto estan aplicant el concepte de "màquina intel-ligent" per poder ampliar l'espai professional.

D'altra banda, estan apareixent plataformes comercials amb aplicació d'IA en àmbits que inclouen la recerca, les capacitats full stack, l'IOT industrial, l'àudio, la visualització i l'enriquiment de dades. I un altre àmbit que comença a apostar fort per la IA és la salut.

En general, tots els àmbits de la indústria tenen curiositat sobre com aprofitar l'aprenentatge automàtic: la publicitat (AdTheorent, Dstillery, Tapad), l'agricultura (BlueRiver, Tule,

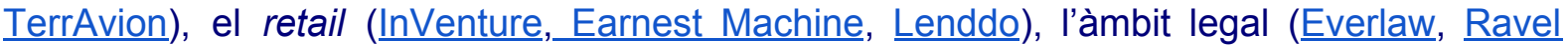
Law, Seal Software), la fabricació (Zyymergen, Ginkgo Bioworks, Sight Machine), el món educatiu (Knewton, Udacity, Gradescope), el transport i la logística (Preteckt, $\underline{\text { ClearMetal, }}$ Nauto) i les finances (Quantopian, Kensho, iSentium) [38].

Apple s'ha unit a Amazon, Google i Facebook per crear un grup de recerca en IA [44], i a Nokia en temes d'eHealth [45]. També està desenvolupament el seu propi altaveu intel-ligent a l'estil d'Amazon Echo o Google Home d'Alphabet.

Val a dir però, que és dins l'àmbit dels dispositius mòbils on es detecta una major implementació de la IA. Alguns exemples són:

- Reconeixement facial. Tant Google [39] com Microsoft [40] estan treballant en l'aplicació d'IA en xips Raspberry per poder implementar sistemes neuronals de manera massiva en dispositius de mida reduïda.

- Millores en navegadors i assistents (Google, Bixby de Samsung), que personalitzen la informació d'entrada ajustada a les prioritats de l'usuari [41] [42]. Un dels productes destacats seria l'Alexa i el seu ús a l'Echo d'Amazon.

- Reconeixement d'imatges a través d'una aplicació que utilitza IA (Google Lens al telèfon Pixel) i altaveus intel-ligents [43].

- Personalització d'un xatbot a través de converses amb l'usuari fins crear-ne una rèplica a través de l'aprenentatge, mitjançant l'App Replika [46] [47]. La premisa de Replika és "Aprèn a obrir-te i ser vulnerable, ensenya Replika a convertir-se en la IA més humana"

A nivell institucional, la Xina és el país que comença a realitzar polítiques i actuacions per posicionar-se clarament al capdavant en la carrera tecnològica a les IA com a gran potència, augmentar la productivitat del país i oferir major poder als treballadors. Part del pla consisteix en dedicar més recursos a promoure el talent, així com utilitzar IA en educació,

\footnotetext{
5 "Unlock your emotional intelligence. Learn to open up and be vulnerable, teach your Replika to become the most human Al".
} 
salut i seguretat. També preveu oferir incentius a aquelles companyies estrangeres que obrin centres I+D al país [48].

En aspectes de seguretat, vam conèixer aquest any que la Xina utilitza la IA per intentar evitar crims abans que succeeixin [49]. La IA s'encarrega d'analitzar les cares de totes les persones a través de la tecnologia de reconeixement facial Cloud Walk que ofereix un sistema que analitza els moviments i comportaments de les persones per avaluar les possibilitats que tenen de cometre un crim poder avisar la policia sobre aquests "criminals potencials".

Dins la branca de la IA que deriva en robòtica, seguint el corrent de l'any anterior, continua present la preocupació pel tema laboral. El motiu és que tasques mecàniques de feines poc qualificades podran ser fàcilment automatitzades per robots però, com ha passat ja en anteriors revolucions industrials, quan desapareixen certs llocs de treball a causa de l'automatització, també s'espera que n'apareguin de nous lligats a aquests avenços i que obrin un nou nínxol laboral [50] [51] [52].

Segons un informe d'ADECCO (consultora de recursos humans), alguns dels llocs de treball amb més demanda, segons les necessitats actuals, són [53]:

1. Analista de Big Data.

2. Híbrids entre disseny i tecnologia: investigador d'usuari, analista d'usabilitat, arquitecte d'informació, dissenyador interactiu o en UX, etc.

3. Psicòlegs i coaches.

4. Cloud computing expert.

6. Metges de fetus (per curar malalties abans del naixement).

7. Professors llicenciats en hackschooling, per a infants que seran nadius digitals.

8. Algunes més futuristes: "ciberassessor" financer, conductor de drons, enginyers industrials especialitzats en robòtica.

Anteriorment ja havíem comentat els debats ètics i legals que genera o, millor dit, que generarà l'ús a petita i gran escala de la robòtica quan hagi arribat a àmbits crucials de la nostra vida diària.

El Parlament Europeu ha elaborat un estudi/proposta i preveu establir un marc legal comú per a la industria de la robòtica [54] que podria incloure els següents punts, en relació als robots:

- Haurien de disposar d'interruptor d'emergència.

- No poden fer mal als humans.

- Es prohibeix establir vincles emocionals amb ells.

- Els de major mida (com ara vehicles) han de tenir assegurança obligatòria.

- Han de tenir drets i obligacions, una figura legal de "persona electrònica".

- Hauran de pagar impostos (cotitzaran a la seguretat social). 
- Es crearà una renda bàsica universal.

Els dos darrers punts estan clarament encarats a posar solució al tema de la mancança de vacants laborals: la renda universal en el cas dels llocs de treball que desapareixerien i el tema de la cotització per tal de mantenir les prestacions i equilibris en els estats. A la pròpia UOC es va publicar una entrada [55] en la qual es debatia qui, si empresaris o fabricants, han de pagar la cotització dels robots.

\section{Conclusions}

Hem vist com en poc temps la IA ha passat de no veure's inclosa en els reports de tendències encapçalar-los. Ja s'estan movilitzant esforços per regular legalment alguns aspectes relacionats amb aquestes tecnologies. Institucions com el Future of Life Institute vetllen per com les màquines intel-ligents estan interactuant amb l'ésser humà i com hem vist, la IA està o estarà, present en tots els àmbits: l'automoció, la salut, la fabricació, el màrqueting, etc. Val la pena destacar la seva presència en disciplines més creatives com l'art, la música i l'entreteniment en general; o fins i tot en educació, on hi ha hagut experiències que mostren que la IA pot ser un suport per professors i estudiants.

Des d'un punt de vista dels beneficis, la IA hauria de permetre fer de forma més ràpida i eficient tasques rutinàries. L'"smartització6" conjugada amb la IA obren la porta a grans avenços dins l'àmbit domèstic gràcies als robots familiars i a la domòtica avançada. Els beneficis d'aquest canvi poden anar des de fer la vida més còmoda a les persones, fins a ajudar a persones amb capacitats reduïdes, ancianes, i amb necessitat d'ajuda.

En salut, la IA està començant a esdevenir un suport en la diagnosi de malalties, tot i que encara s'està lluny de substituir el metge. També en l'àmbit jurídic hi ha hagut experiments per avaluar com es comportaria una IA com a jutge.

Des d'un punt de vista negatiu, la IA presenta riscos com la pèrdua de llocs de treball en ser substituïts per les màquines, vigilància $24 / 7$, captació de dades que escapen al nostre control, armes intel-ligents, creació d'una societat alienada pels bots conversacionals, etc. Tot i així, s'està treballant per tal que, almenys des d'un punt de vista legislatiu, aquests riscos quedin minimitzats.

En el punt actual, sembla que la IA es troba en procés de maduració: està mostrant moltes possibilitats però caldrà veure quines cristal-litzen, i com, els propers anys.

\footnotetext{
${ }^{6}$ Fem referència aquí al concepte smart i com aquesta tecnologia ha entrat en els diferents terrenys dels aparells electrònics que emprem diàriament, és a dir, convertir en intel-ligent o dotar de característiques tecnològiques avançades tant televisions com telèfons, vehicles i altres objectes o processos.
} 


\section{Infografia}

\section{Previsions i realitats de la Intel-ligència Artificial Evolució 2015-2017}

\section{Previsions}

- IA no contemplada com a tendència principa

- Augment anual del mercat global de màquines intel-ligents $>19,7 \%$ el 2019

- Futur IA: Google, Apple, Facebook, Amazon, IBM, Baidu, Microsoft, Yahoo

$$
\text { గि }
$$$$
2015
$$

- Medicina · Educació

- Llar · Mobilitat ·

- Àmbit laboral

- Assistents personals

\section{Realitats}

- Carta oberta contra l'ús d'armes autònomes

- Alpha Go (Google) venç al campió mundial

- Cotxes autònoms com a realitat comercial

- DARPA Robotics Challenge

- Pensament

abstracte creatiu.

Xarxes neuronals .

2015

cloud computing.
- IA dins les 6 tecnologies que canviaran el món Google

- Tendència capdavantera per al 2017 Forrester

- IA com a tecnologia emergent puntera CIO New Year's Resolutions 2016

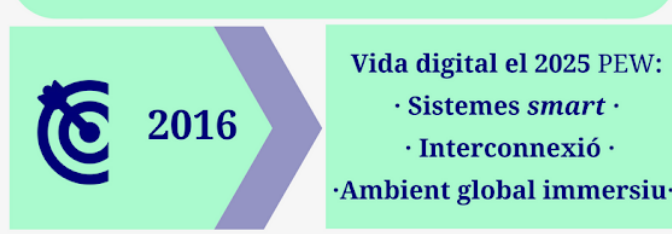

- Alarmes socials: robots, noves professions

- Regulació robòtica i ètica

- Interacció humà-màquina i Machine Learning

- IBM Watson respon en llenguatge natural

- Robots professors de robots

- Diagnòstics mèdics ·

- Assistents personals

- Dispositius mòbils

vehicles, drones .
2016

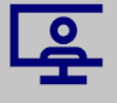

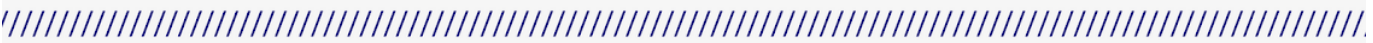

- Xatbots

- Més confiança en la IA i ètica

- Estàndards universals

- Deep Reinforcement Learning

- Models predictius amb Big Data

- Interació home-màquina (intel. augmentada)

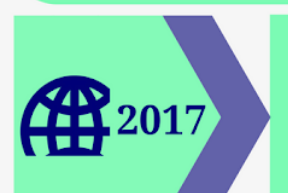

- Robòtica · IOT ·

- Aprenentatge automàtic ·

- Deep Learning ·
- Projecte PAIR (Google, MIT, Harvard)

- Mòbils: millores assistents, reconeixement imatges

- Bots conversacionals avançats (App Replika)

- IA seguretat (prevenció de crims)

- Substitució laboral per robots, noves professions

- Marc legal indústria robòtica (Parlament Europeu)

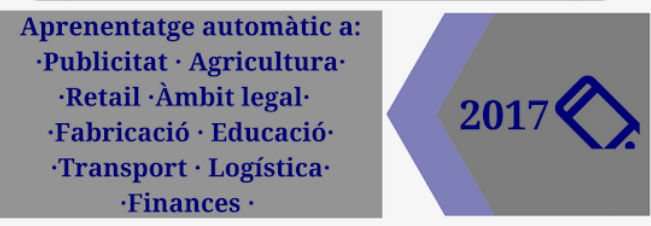
Finances .

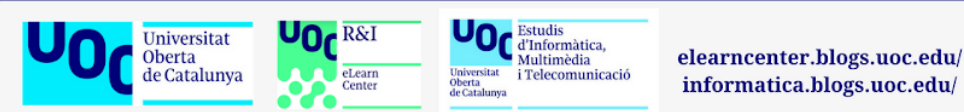




\section{Referències}

[1] Allison G. S. et alii, "Tech Trends 2015," Frog Design, 2015. Disponible a https://www.frogdesign.com/html/techtrends2015/index.html. [Data de consulta 16/05/2018]. [2 ] Neira, E. M., "IEEE ComSoc CTN Special Issue on Ten Trends that Tell Where Communication Technologies are Headed in 2015," IEEE CTN, 2015. Disponible a https://www.comsoc.org/ctn/ieee-comsoc-ctn-special-issue-ten-trends-tell-where-communica tion-technologies-are-headed-2015 [Data de consulta 16/05/2018].

[3] Chokshi, S., Horowitz, A., "16 Things", 2015, Andresseen Horowitz. Disponible a https://a16z.com/2015/01/22/16-things/. [Data de consulta 16/05/2018].

[4] "10 Breakthrough Technologies 2015", MIT Technology Review, 2015. Disponible a https://www.technologyreview.com/lists/technologies/2015/ [Data de consulta 16/05/2018].

[5] "Baròmetre del Sector tecnològic a Catalunya", Cercle Tecnològic de Catalunya, Barcelona, 2014.2 Disponible a http://www.ctecno.cat/wp-content/uploads/2014/07/TECHNOLOGICAL-SECTOR-BAROMET ER-IN-CATALONIA-2014-ENGLISH.pdf [Data de consulta 16/05/2018].

[6] Spender, A., "Top 10 Strategic Predictions for 2015 and Beyond", Gartner, 2015. Disponible

https://www.gartner.com/smarterwithgartner/top-10-strategic-technology-predictions-for-2015 -and-beyond/ [Data de consulta 16/05/2018].

[7] "Autonomous weapons: an open letter from Al \& robotics researchers", Future of life, 2015. Disponible a http://futureoflife.org/open-letter-autonomous-weapons/. [Data de consulta 16/05/2018].

[8] Hassabis, D., "AlphaGo: using machine learning to master the ancient game of Go", Google Official Blog. Machine Learning, 2015. Disponible a https://googleblog.blogspot.co.il/2016/01/alphago-machine-learning-game-go.html [Consultat el 16/05/2018].

[9] Asendador, S. H., "Seis tecnologías que cambiarán el mundo según Google", Expansión. Economía Digital, 2016.2 Disponible a http://www.expansion.com/economia-digital/innovacion/2016/06/11/575c094b22601d01628b 4611.html [Consultat el 25/04/2018].

[10] Alkhatib, H. et al., "IEEE CS 2022 Report", IEEE.org, 2014. Disponible a http://lifesciences.ieee.org/article-archive/ieee-cs-2022-report/. [Consultat el 22/05/2018].

[11] Anderson, J., "Digital Life in 2025", Pew Research Center, 2014. Disponible a http://www.pewinternet.org/2014/03/11/digital-life-in-2025/ [Consultat el 16/05/2018].

[12] Raskino M., Meehan P., "CIO New Year's Resolutions 2016", Gartner, 2016. Disponible ahttps://www.gartner.com/doc/3182825/cio-new-years-resolutions-. [Consultat el 16/05/2018].

[13] Hopkins, B., "Forrester's top emerging technologies to watch in 2017-2021", Forrester, 2016.

Disponible 
https://go.forrester.com/blogs/16-09-14-forresters_top_emerging_technologies_to_watch_20 17 2021/. [Consultat el 22/05/2018].

[14] Yuhas, A., "Would you bet against sex robots? Al 'could leave half of world unemployed" The Guardian, 2016.

Disponible a https://www.theguardian.com/technology/2016/feb/13/artificial-intelligence-ai-unemploymentjobs-moshe-vardi. [Consultat el 22/05/2018].

[15] Garfield, L., "A 19-year-old made a free robot lawyer that has appealed \$3 million in parking tickets" Business Insider, 2016.Disponible a http://www.businessinsider.com/joshua-browder-bot-for-parking-tickets-2016-2. [Consultat el 22/05/2018].

[16] Slayter, M. E., "11 Really Cool Jobs That Don't Exist Today, But Will Soon", Fortune.com, 2016. Disponible a http://fortune.com/2016/02/16/jobs-future/. [Consulta el 22/05/2018].

[17] Elola, J. "El futuro de la humanidad en sus manos", El País, 2016. Disponible a https://elpais.com/elpais/2016/02/12/ciencia/1455304552_817289.html. [Consultat el 22/05/2018].

[18] Bland, J., "A ban on autonomous weapons is easier said than done", The Guardian, 2015.

Disponible

a

https://www.theguardian.com/science/political-science/2015/jul/31/artificial-intelligence-ban-a utonomous-weapons-easier-said-than-done. [Consultat el 22/05/2018].

[19] Hodgkins, K., "Facebook's Al is Learning Language Via Children's Books", Digital Trends, 2016.

Disponible

https://www.digitaltrends.com/cool-tech/facebooke-ai-children-books/. [Consultat el 22/05/2018].

[20] P. Bright, "Microsoft terminates its Tay Al chatbot after she turns into a Nazi", Ars 2016.

https://arstechnica.com/information-technology/2016/03/microsoft-terminates-its-tay-ai-chatb ot-after-she-turns-into-a-nazi/. [Consultat el 28/05/2018].

[21] Margolin, M., "These Glasses Fool Facial Recognition Into Thinking You're Someone Else", $\quad$ Motherboard, 2016.2 Disponible a https://motherboard.vice.com/en_us/article/pgkxgv/glasses-fool-facial-recognition [Consultat el 28/05/2018].

[22] Summers, N., "People will lie to robots to avoid hurting their feelings," Engadget, 2016. Disponible a https://www.engadget.com/2016/08/24/people-lie-robots-avoid-hurting-feelings/ . [Consultat el 28/05/2018].

[23] Clarisó R., et al., "Informe de l'Observatori Tecnològic dels Estudis d'Informàtica, Multimèdia i Telecomunicació", Estudis d'Informàtica, Multimèdia i Telecomunicació, Universitat Oberta de Catalunya, 2017. Disponible a http://openaccess.uoc.edu/webapps/o2/bitstream/10609/80806/6/informeobservatori2017pu blic.pdf [Consultat el 28/05/2018].

[24] Jozuka, E., "A Japanese Al Almost Won a Literary Prize", Motherboard, 2016. Disponible

https://motherboard.vice.com/en_us/article/wnxnjn/a-japanese-ai-almost-won-a-literary-prize. [Consultat el 28/05/2018]. 
[25] Bourzac, K. "Bringing Big Neural Networks to Self-Driving Cars, Smartphones, and Drones", IEEE Spectrum, $2016 . \quad$ Disponible a http://spectrum.ieee.org/computing/embedded-systems/bringing-big-neural-networks-to-selfd riving-cars-smartphones-and-drones. [Consultat el 28/05/2018].

[26] Twilley, N., "Say What You See, Facebook," New Yorker, 2016. Disponible a http://www.newyorker.com/tech/elements/say-what-you-see-facebook. [Consultat el 28/05/2018].

[27] Metz, C., "How Google's Al Viewed the Move No Human Could Understand", Wired, 2016; Disponible a https://www.wired.com/2016/03/googles-ai-viewed-move-no-human-understand/. [Consultat el 28/05/2018]. Veure també Atwood, J., "Thanks For Ruining Another Game Forever, Computers,", Coding horror, $2016 . \quad$ Disponible $\quad$ a https://blog.codinghorror.com/thanks-for-ruining-another-game-forever-computers/.

[Consultat el 28/05/2018].

[28] Oberhaus, D., "This Robotic Centipede Will Teach Robots How to Walk" Motherboard, 2016.

https://motherboard.vice.com/en us/article/z43y38/robotic-centipede-walking. [Consultat el 28/25/2018].

[29] Vincent, J., "Satya Nadella's rules for Al are more boring (and relevant) than Asimov's Three Laws", The Verge, 2016. Disponible a http://www.theverge.com/2016/6/29/12057516/satya-nadella-ai-robot-laws. [Consultat el 28/05/2018].

[30] Metz, C., "Artificial Intelligence Is Setting Up the Internet for a Huge Clash With Europe" Wired, $2016 . \quad$ Disponible

https://www.wired.com/2016/07/artificial-intelligence-setting-internet-huge-clash-europe/.

[Consultat el 28/05/2018].

[31] Vincent, J.,"First Click: Deep learning is creating computer systems we don't fully understand", The Verge, 2016. Disponible http://www.theverge.com/2016/7/12/12158238/first-click-deep-learning-algorithmic-black-box es. [Consultat el 28/05/2018].

[32] Jagadish, H., "Algorithms can be more fair than humans," The Conversation, 2016. Disponible a https://theconversation.com/algorithms-can-be-more-fair-than-humans-64047. [Consultat el 28/05/2018].

[33] Stroud, M., "Chicago's predictive policing tool just failed a major test," The Verge, 2016. Disponible

http://www.theverge.com/2016/8/19/12552384/chicago-heat-list-tool-failed-rand-test.

[Consultat el 28/05/2018].

[34] Templeton, G., "Al beats doctors at visual diagnosis, observes many times more lung cancer signals", ExtremeTech, 2016. Disponible a http://www.extremetech.com/extreme/233746-ai-beats-doctors-at-visual-diagnosis-observesmany-times-more-lung-cancer-signals. [Consultat el 28/05/2018].

[35] "Gartner Top 10 Technology Trends 2017", Mavim, 2017. Disponible a https://www.youtube.com/watch?v=fTvFO-2LtNM. [Consultat el 28/05/2018].

[36] Gatherer, A., "Ten Communications Technology Trends for 2017", IEEE Communications Society, $2017 . \quad$ Disponible 
http://www.comsoc.org/ctn/ten-communications-technology-trends-2017. [Consultat el 28/05/2018].

[37] Hidalgo, F. J., "Google lanza nuevo proyecto para mejorar la capacitación de la Inteligencia Artificial," Wwwhat's new, 2017. Disponible a https://wwwhatsnew.com/2017/07/10/google-lanza-nuevo-proyecto-para-mejorar-la-capacita cion-de-la-inteligencia-artificial/. [Consultat el 28/05/2018].

[38] Nichol, P. B., "3 things to know before launching Machine intelligence", CIO, 2017. Disponible

https://www.cio.com/article/3191511/artificial-intelligence/3-things-to-know-before-launchingmachine-intelligence.html. [Consultat el 28/05/2018].

[39] Lomas, N., "Google Planning Al tools for Pi makers this year", TechCrunch, 2017. Disponible

https://techcrunch.com/2017/01/25/google-planning-ai-tools-for-pi-makers-this-year/.

[Consultat el 28/05/2018].

[40] Álvarez, R., "Microsoft ha logrado meter redes neuronales en este pequeño chip que es compatible con Raspberry Pi," Xataka, 2017. Disponible a https://m.xataka.com/makers/microsoft-ha-logrado-meter-redes-neuronales-en-este-pequen o-chip-que-es-compatible-con-raspberry-pi. [Consultat el 28/05/2018].

[41] Gibbs, S., "Google to radically change homepage for first time since 1996", The 2017.

Disponible https://www.theguardian.com/technology/2017/jul/20/google-change-home-page-first-time-si nce-1996-app-based-feed-news-events-sports. [Consultat el 28/05/2018].

[42] Álvarez, R., "Bixby y cómo quiere ganar Samsung la guerra de los asistentes con inteligencia artificial”, Xataka, 2017. Disponible a https://m.xataka.com/robotica-e-ia/bixby-y-como-quiere-ganar-samsung-la-guerra-de-los-asi stentes-con-inteligencia-artificial. [Consultat el 28/05/2018].

[43] Wakabayashi, D., "Google's New Gadgets Come With a Big Helping of A.I.", NYTimes.com, $2017 . \quad$ Disponible https://mobile.nytimes.com/2017/10/04/technology/google-pixel-hardware.html?referer=.

[Consultat el 28/05/2018].

[44] Steele, B., "Apple joins Amazon, Google and Facebook in Al research group," Engadget, 2017.2 Disponible a https://www.engadget.com/2017/01/27/apple-joins-partnership-on-ai/. [Consultat el 28/05/2018].

[45] M. Carrol, "Nokia, Apple draw line under latest patent spat" Mobile World Live, 2017. Disponible

https://www.mobileworldlive.com/devices/news-devices/nokia-apple-draw-line-under-latest-p atent-spat/. [Consultat el 28/05/2018].

[46] Pardes A., "What my personal chatbot Replika is teaching me about Artificial Intelligence", $\quad$ Wired, $2017 . \quad$ Disponible a https://www.wired.com/story/what-my-personal-chat-bot-replika-is-teaching-me-about-artifici al-intelligence/?mbid=nl 111217 daily list3_p3 [Data de consulta 16/05/2018].

[47] Murphy M., Templin J., "This app is trying to replicate you", Quartz, 2017. Disponible a https://classic.qz.com/machines-with-brains/1018126/lukas-replika-chatbot-creates-a-digital-r epresentation-of-you-the-more-you-interact-with-it/. [Data de consulta 16/05/2018]. 
[48] Domenech, J., "China estrenará un plan nacional enfocado en la Inteligencia Artificial", Silicon, $2017 . \quad$ Disponible a https://www.silicon.es/china-estrenara-plan-nacional-enfocado-la-inteligencia-artificial-23445 02. [Data de consulta 15/05/2018].

[49] Araújo S., "China utiliza una inteligencia artificial para intentar evitar crímenes antes de que sucedan," Genbeta, 2017. Disponible a https://m.genbeta.com/actualidad/china-utiliza-una-inteligencia-artificial-para-intentar-evitar-c rimenes-antes-de-que-sucedan. [Data de consulta 15/05/2018].

[50] Moya, P., "Descubre si un robot te va a quitar el trabajo con esta web," Omicrono, 2017. Disponible a http://omicrono.elespanol.com/2017/05/web-robot-te-va-a-quitar-el-trabajo/. [Data de consulta 15/05/2018].

[51] "Les sept métiers que les robots intelligents vont nous prendre en premier" Slate, 2017. Disponible http://www.slate.fr/story/144801/sept-metiers-robots-intelligents-prendre-en-premier. [Data de consulta 15/05/2018].

[52] "La robotización creará dos millones de empleos netos hasta el 2030," El Periódico, 2017.

Disponible

http://www.elperiodico.com/es/economia/20170718/la-robotizacion-creara-dos-millones-de-e mpleos-netos-hasta-el-2030-en-espana-6174198. [Consultat el 28/05/2018].

[53] Lorente, S., "Estos serán los trabajos más populares del futuro," Harper's Bazaar, 2017. Disponible https://www.harpersbazaar.com/es/cultura/viajes-planes/a356884/trabajos-futuro-revolucionindustrial/. [Consultat el 28/05/2018].

[54] "Delvaux propone normas europeas para la robótica y un seguro obligatorio", Parlamento Europeo, 2017. Disponible a http://www.europarl.europa.eu/news/es/headlines/economy/20170109STO57505/delvaux-pr opone-normas-europeas-para-la-robotica-y-un-seguro-obligatorio. [Consultat el 28/05/2018]. [55] Bigas, N., "Empresaris o fabricants, qui ha de pagar la cotització dels robots?", UOC News, 2017. https://www.uoc.edu/portal/ca/news/actualitat/2017/256-robots-cotitzacio.html. [Consuñtat el 26/04/52018].

[56] "The Top A.I. Breakthroughs of 2015", Future of Life, 2015. Disponible a: https://futureoflife.org/2015/12/29/the-top-a-i-breakthroughs-of-2015/. [Consultat el 28/05/2018].

[57] Clark, J., "Why 2015 Was a Breakthrough Year in Artificial Intelligence", Bloomberg, 2015.

https://www.bloomberg.com/news/articles/2015-12-08/why-2015-was-a-breakthrough-year-in -artificial-intelligence. [Consultat el 28/05/2018].

[58] Knight, W., "What Robots and Al Learned in 2015", MIT Technology Review, 2015. Disponible

https://www.technologyreview.com/s/544901/what-robots-and-ai-learned-in-2015/. [Consultat el 28/05/2018].

[59] Knight, W., "DARPA's Robot Challenge May Equip Robots to One Day Walk Among Us" MIT Technology Review, 2015.2 Disponible 
https://www.technologyreview.com/s/537931/darpas-robot-challenge-may-equip-robots-to-on e-day-walk-among-us/. [Consultat el 28/05/2018].

[60] McWilliams, A. "Smart Machines: Technologies and Global Markets", BCC Reseach, 2014. Citat a "The Forecast. Artificial Intelligence", Usbek \& Rica, 2015. Presentació en format Slideshare disponible a https://www.slideshare.net/usbeketrica/the-forecast-artificial-intelligence-55572992.

[Consultat el 30/05/2018].

[61] Op. cit. (Usbek \& Rica, 2015).

[62] Lant, K. "Al forecast for 2017", Futurism, 2017. Infografia disponible a https://futurism.com/images/ai-forecast-for-2017/. [Consultat el 30/05/2018].

[63] "Artificial Intelligence", English Oxford Living Dictionary. Disponible a https://en.oxforddictionaries.com/definition/artificial intelligence. [Consultat el 15/04/2018].

[64] McCarthy, J. et al., "Dartmouth Al Project Proposal", 1955. Disponibles a http://www-formal.stanford.edu/jmc/history/dartmouth/dartmouth.html. [Consultat el 15/04/2018].

[65] Penrose, R. "The Emperor's New Mind: Concerning Computers, Minds and The Laws of Physics”, Oxford University Press, 1989.

[66] Ross, C., Swetlitz, I., "IBM pitched its Watson supercomputer as a revolution in cancer care. It's nowhere close", Stat, 2017. Disponible a https://www.statnews.com/2017/09/05/watson-ibm-cancer/. [Consultat el 25/06/218].

[67] Auchard, E., "Artificial intelligence poses risks of misuse by hackers, researchers say", Reuters, 2018.

Disponible

https://www.reuters.com/article/us-cyber-tech/artificial-intelligence-poses-risks-of-misuse-byhackers-researchers-say-idUSKCN1G503V. [Consultat el 26/06/218].

[68] Chennu, S., "DeepMind: can we ever trust a machine to diagnose cancer?" The Conversation, 2017.2 Disponible a http://theconversation.com/deepmind-can-we-ever-trust-a-machine-to-diagnose-cancer-8870 7. [Consultat el 26/06/218].

[69] Per a tal efecte es poden consultar llibres com ara la següent referència: Winston, P.H., "Artificial Intelligence", Massachusetts Institute of Technology, 1992.

[70] Veure nota anterior. Russell, S., Norvig P., "Artificial Intelligence: A Modern Approach", 1995; 3rd. ed. 2017, Pearson.

[71] Corea, F. "Artificial Intelligence: What is It and Why Now?" Datafloq, 2018. Disponible a https://datafloq.com/read/artificial-intelligence-what-is-it-why-now/2471. [Consultat el 25/06/218]. 